\title{
Prostate Cancer Treatment on the Basis of an Individual Risk Profile; Can we Reduce Overtreatment?
}

\author{
Eelco R.P. Collette* and Monique J. Roobol
}

\author{
Department of Urology, Erasmus University Medical Center, P.O. Box 2040, 3000 CA Rotterdam, The \\ Netherlands
}

\begin{abstract}
Prostate cancer $(\mathrm{PCa})$ is the most prevalent cancer in male population with an incidence rate of 93 per 100.000 men in Europe and is the sixth leading cause of cancer related deaths in men. In the last two decades the incidence of $\mathrm{PCa}$ has increased, which is related to widespread prostate-specific antigen (PSA) based screening and increased life expectancy. Mortality rates of prostate cancer have been reduced due to improvement in treatment and/or the widespread screening activities. Major down sides of screening are the potential risks of overdiagnosis and subsequent overtreatment. Approximately $50 \%$ of PCa cases detected through screening are potentially overdiagnosed and hence do not require active treatment. However, in clinical practice men with a potentially non-life-threatening cancer (indolent cancer) are often treated actively resulting in unnecessary suffering from serious side effects coinciding with active treatment. The way out of this dilemma is two-fold. First, the actual diagnosis could be delayed or even avoided and second, radical treatment could be delayed or avoided for patients with low-risk PCa. To better predict the presence of a (potentially indolent) prostate cancer nomograms have been developed. These multivariate prediction tools can be of aid in avoiding unnecessary biopsies reducing overdiagnosis, or identifying potentially indolent prostate cancer after diagnosis and hence adapt the treatment strategy. In this expert opinion we discuss the available tools and their performance in reducing the unwanted side effects of prostate cancer screening. In addition, we provide an overview of strategies concerning optimisation and individualisation of treatment, to reduce overtreatment of prostate cancer.
\end{abstract}

Keywords: Prostate cancer, indolent disease, PSA, screening, mortality reduction, overdiagnosis, overtreatment, comorbidity, prediction tool, nomogram, risk calculator.

\section{INTRODUCTION}

Prostate cancer $(\mathrm{PCa})$ is a major health problem. It is the most prevalent cancer in male population. The last two decades incidence rates have increased rapidly. With an incidence rate of 93 per 100.000 men in Europe it is the sixth leading cause of cancer related deaths in men [1]. The increased incidence of $\mathrm{PCa}$ is related to widespread prostate-specific antigen (PSA) based screening, increased awareness of $\mathrm{PCa}$ and increased life expectancy [2]. In 2008 over 258.000 deaths were related to PCa globally [1].

The suspicion of the presence on PCa is based on several tests. In general a digital rectal examination (DRE), transrectal ultrasound (TRUS) and serum prostate specific antigen (PSA) testing are used to decide whether a prostate biopsy is indicated. At the moment the ultrasound guided prostate biopsy is still considered the "gold standard" in the diagnosis of PCa. Previously, often in response to an abnormal DRE a biopsy was performed; today PSA determination is usually the cause.

\footnotetext{
*Address corresponding to this author at the Department of Urology, Erasmus University Medical Center, P.O. Box 2040, 3000 CA Rotterdam, The Netherlands; Tel: 003110-7034548; Fax: 003110-7035315;

E-mail: eelco@collette.nl
}

Due to the increased screening of $\mathrm{PCa}$ in the current PSA era the incidence of the disease has almost doubled in the last twenty years [2]. Mortality rates of $\mathrm{PCa}$ are declining most likely due to improvement in treatment and/or the widespread screening activities [3, 39]. The European Randomised Study for Screening of Prostate Cancer (ERSPC) concluded that systematic PSA-based screening for prostate cancer of the general population reduces prostate cancer specific mortality by at least $20 \%$ [4]. The Goteborg trial (part of ERSPC) showed that prostate cancer mortality was reduced by almost $50 \%$, applying a two year screening interval and having the availability of 14 years of follow-up [5].

Major down sides of screening are the potential risks of overdiagnosis and subsequent overtreatment $[4,5]$. As PSA screening increases, more patients without any clinical symptoms are diagnosed with $\mathrm{PCa}$, which possibly would never become clinically apparent [2]. It is estimated that approximately $50 \%$ of $\mathrm{PCa}$ cases detected through screening are potentially overdiagnosed and hence do not require active treatment [2]. However in clinical practice men with a potentially non-life threatening cancer (indolent cancer) are often treated actively resulting in unnecessary suffering from serious side effects coinciding with active treatment. Over $90 \%$ of men with PSA-detected prostate cancer undergo early treatment and less than 
$7 \%$ elect for active surveillance in the United States [6]. The introduction of PSA screening has resulted in the United States in more than 1 million additional men being diagnosed and treated [7]. Active treatment options are associated with adverse outcomes; after prostatectomy or external radiotherapy, erectile dysfunction and urinary incontinence may occur. Urinary incontinence was observed in $14 \%$ and $31 \%$ after surgery and in $4 \%$ and $13 \%$ after radiotherapy; erectile dysfunction was observed in $79 \%$ and $88 \%$ after surgery and in $63 \%$ and $64 \%$ after radiotherapy $[8,9]$. Maintaining quality of life after active treatment is an important issue. A Scandinavian randomized trial compared radical prostatectomy to watchful waiting and showed no difference in any psychological domain between the watchful waiting and surgery group [10]. Quality of life data showed that negative side effects of both strategies were common and that both interventions added more stress than would be seen in a background population. In the prostatectomy group, urinary leakage and erectile dysfunction were often consequences of surgery, whereas in the watchfulwaiting group, they were due to tumor progression and/or hormone treatment. Most side effects concerning the urinary tract improved after some years, but substantial symptoms persisted in many patients up to 5 years after treatment. They reported lower physical functioning 5 to 10 years after treatment than the control group of similar age [10]. In a study using a computer model as a virtual laboratory for translational research based on the results from one of the leading active surveillance cohorts, the authors concluded that active surveillance among men diagnosed with low-risk prostate cancer could lead to significant benefits in terms of quality of life comparing to men treated with immediate radical prostatectomy [45].

Additional methods are desirable to identify men with a potentially life threatening cancer or vice versa to identify those without a potentially life threatening cancer. This will decrease the adverse effects of screening. The way out of this dilemma is two-fold. First the actual diagnosis could be delayed or even avoided and second, radical treatment could be delayed or avoided for patients with low-risk PCa.

In this expert opinion we discuss the available prediction tools and their performance in reducing unwanted prostate biopsies and overdiagnosis. In addition, we provide an overview of strategies concerning optimisation and individualisation of treatment, to reduce overtreatment of prostate cancer.
PREDICTION TOOLS; REDUCING BIOPSIES AND OVERDIAGNOSIS

To better predict the presence or outcome of disease, prediction tools have been developed. They are being used increasingly in modern medicine [14]. A recent literature review identified $>100$ predicting models for prostate cancer outcome [11, 12]. These multivariate prediction models and nomograms may be helpful in shared-decision making [13]; they can be of aid in avoiding unnecessary biopsies resulting in the diagnosis of a potentially indolent cancer or to identify potentially indolent prostate cancer after diagnosis and hence adapt the treatment strategy. The current tests (DRE, TRUS and serum-PSA) for predicting the presence of $\mathrm{PCa}$ in an individual patient, have their specific weaknesses and strengths. Other risk factors can be helpful, such as family history, age, prostate volume and prior negative biopsies. Combining tests might help to increase the predictive capability. By including all these factors into a prediction model the likelihood of a biopsy detectable (potentially aggressive) PCa can be assessed which can be helpful in the question whether or not to perform a prostate biopsy [11].

The reason for this consideration is that a prostate biopsy is not a harmless procedure, minor side effects are more common than major side effects; haematuria and haemospermia occur in $22.6 \%$ and $50.4 \%$ of men biopsied, respectively [42]. Major side effects were studied in a large European screening cohort; fever and hospital admission were reported in $4.2 \%$ and $0.8 \%$, respectively. Although most fevers were managed on an outpatient basis, $81 \%$ of hospital admissions were for infection [43].

In addition, to simply predict biopsy outcome (having PCa yes or no) some risk calculators may help us discriminate potentially low risk cancer from aggressive cancer and estimate the risk of progression. Hence these different predicting tools exist throughout the path from screening to palliative treatment.

\section{TOOLS PREDICTING BIOPSY OUTCOME}

There are three commonly known online risk calculators predicting the presence of a biopsy detectable PCa. The North-American Prostate Cancer Prevention Trial (PCPT) risk calculator [15] is based on a study evaluating a possible preventive effect of Finasteride in PCa development and includes serumPSA, outcome of DRE, prior biopsy, race, age and family history. Data was obtained from over 5500 
males from the placebo group and these were used to create the risk calculator. There was a reported AUC of 0.70 for the calculator in the original study, which was higher than the 0.68 reported for PSA alone [16]. It has been validated in external populations, with accuracies ranging from 0.57 to 0.74 [17-19].

The second is the Sunnybrook risk calculator which combines a variety of variables like DRE, PSA, percent free PSA, age, ethnicity, family history of $\mathrm{PCa}$ and urinary voiding symptom score [20]. It is derived using a clinical cohort of 3100 men. It achieved an AUC of 0.74 for any PCa and 0.77 for high-grade cancer. This was significantly greater than the conventional screening method of DRE and PSA only, which was 0.62 for any cancer and 0.69 for high-grade cancer [21]. As was demonstrated in a prospective head-tohead comparison in more than 2100 patients who underwent a prostate biopsy, the Sunnybrook calculator outperformed the PCPT model (AUC 0.67 vs. 0.61 for any cancer; 0.72 vs. 0.67 for predicting aggressive disease) [22]. However, decision curve analysis demonstrated that neither calculator was of clinical benefit, because it did not show a probability threshold considered acceptable with respect to saving biopsies and missing PCa [22].

The third risk calculator is from the European Randomized Study of Screening for Prostate Cancer (ERSPC), which has developed its own online prediction models based on data from the Rotterdam cohort consisting of 6200 Dutch males randomised to the screening arm and aged 55-74 years at time of screening in ERSPC [23, 25]. The risk calculator contains six different steps. According to age, PSA, family history, LUTS, DRE, prostate volume, transrectal ultrasound (presence or absence of hypoechogenic lesions) and prior biopsy information the chance of a negative of positive biopsy and an indolent or aggressive PCa can be calculated. Step 1 and 2 are meant for lay men and GP's and use readily available information (age, LUTS, family history and PSA) to calculate a rough estimate on the chance of having a biopsy detectable PC. Step 3 estimates the chance of a positive prostate biopsy in previously unscreened men and step 4 in previously screened and (optionally) biopsied men using additional information from the outcome of PSA, DRE and TRUS [24, 25]. A promising tool for reducing overdiagnosis of $\mathrm{PCa}$ is a fifth risk calculator which has been developed to predict potentially indolent PCa using PSA, TRUS assessed prostate volume and biopsy information; namely Gleason score and tumor involvement [26]. Screening on the basis of an individual risk assessment using these risk calculators can result in a considerable reduction of unnecessary biopsies and only few important $\mathrm{PCa}$ cases (for which diagnosis at a subsequent screening visit might be too late for treatment with curative intent) would be missed [24]. Applying risk calculator 5 and using a cut-off of $>70 \%$ probability of having a potentially indolent $\mathrm{PCa}$ for the practice of a conservative form of treatment (active surveillance), about $6 \%$ of non-indolent tumours are going to be considered as indolent, and only until they are recognised as a significant tumour, they will be treated as indolent cancers [26]. Step 6 is the latest in the series of prediction tools; it calculates the risk of $\mathrm{PCa} 4$ years after an initially negative screen. It is based on age, PSA, DRE, family history, prostate volume, and previous biopsy status [44]. It predicts an individual's 4-year risk of developing the disease and facilitates stratification of risk, predicting the chance on low- and high-risk PCa, and as such can be of aid in planning future PSA test and/or re-biopsy.

In a head-to-head comparison of the ERSPC and PCPT online nomograms for prostate biopsy outcome prediction, the accuracy of these risk calculators was compared in a specific Portuguese population. Both tools were confirmed to be superior to PSA alone. The ERSPC displayed a $7.96 \%$ increase in the predictive accuracy compared to the PCPT $(77.9 \%$ vs. $69.9 \%$ respectively) ( $p=0.002)$ [27]. In two other head-to-head comparisons, the ERSPC risk calculator again outperformed the PCPT model [28, 29]. The ERSPC risk calculator (AUC: 0.71 ) was superior to the PCPT model (AUC: 0.63 ) and PSA (AUC: 0.55), which was showed after validation in referred patients from a North American cohort [29]. In addition, the ERSPC calculator has been validated in the Finnish and Swedish cohort of the ERSPC [30]. It discriminated well between men with and without PCa among initially screened men, but overestimated the risk of a positive biopsy. External validation of the PCPT calculator across 10 international cohorts revealed varying degree of success highly dependent on the cohort, most likely due to different criteria for and work-up before biopsy; AUCs ranged from a low of $56 \%$ to a high of $72 \%$ in the ERSPC cohorts and were statistically significantly higher than that of PSA in 6 out of the 10 cohorts [46]. There was limited to no net benefit to using the PCPT calculator for biopsy referral compared to biopsying all or no men in all five ERSPC cohorts and benefit within a limited range of risk thresholds in all other cohorts [46]. 
The external validity of the ERSPC risk calculator was assessed in a contemporary clinical cohort. This prospective validation in 320 biopsied Dutch men, with no previous prostate biopsy, showed that the calculator predicted a positive biopsy better than a model with only PSA and digital rectal examination, AUC 0.77 and 0.71 , respectively [47].

In contrast to validations, compliance studies are rarely performed. A study among 443 patients showed that compliance of both patients and urologists with the ERSPC risk calculator recommendation was $83 \%$; in $96 \%$ of cases with biopsy recommendation, patients complied, while $36 \%$ of patients with a negative biopsy advice were actually biopsied [48].

A recent paper proposes a new standard for prostate cancer testing, on the basis of risk-adjusted screening guidelines, with the aim of defining those subgroups of men that most stand to benefit from prostate cancer screening [55].

In summary, in men with a known PSA, risk calculators may hold the promise to identify those who are at increased risk of having PCa and are therefore candidates for biopsy. The risk calculators described above have been validated in external populations and screening cohorts with varying results. Active implementation into daily clinical practice shows encouraging results, although currently no empirical data exists. Further research in this field including long term follow-up is needed.

\section{ACTIVE SURVEILLANCE; STRATEGIES TO AVOID OVERTREATMENT}

Active surveillance (AS) is a strategy that aims to individualise therapy by selecting only those men with significant cancers for curative therapy. It consists of actively monitoring the disease according to a protocol with PSA tests, DRE and prostate (re-)biopsies [35]. The most commonly used criteria for AS are prostatespecific antigen $\leq 10.0 \mathrm{ng} / \mathrm{ml}$, PSA-density $<0.2 \mathrm{ng} / \mathrm{ml}$ per $\mathrm{ml}$, stage $\mathrm{T} 1 \mathrm{C} / \mathrm{T} 2$, Gleason score $\leq 3+3=6$, and $\leq 2$ positive biopsy cores [49]. Patients with favourable tumour characteristics are closely monitored using PSA tests and repeat prostate biopsies. The choice between continued observation and radical treatment is based on disease progression, often defined in terms of the PSA doubling time and/or "upgrading" at repeat biopsy [49]. The aim of active surveillance is to postpone or even avoid invasive therapy, like radical prostatectomy or external radiotherapy in men with low risk disease.
Invasive therapy is indicated, when the tumour shows progression during monitoring [36].

AS has become a widely accepted management strategy, especially for older men with low grade $\mathrm{PCa}$ and low volume disease [31]. A retrospective study in which outcome measures in men with screen-detected $\mathrm{PCa}$ that fit the prementioned criteria and who were managed expectantly, showed a favourable $\mathrm{PCa}$ specific prognosis, after 10 years of follow-up, no $\mathrm{PCa}$ specific mortality was reported, whereas $23 \%$ already has died of other causes [50]. Clinical results of longterm follow-up in large prospective active surveillance cohorts show low rates of prostate cancer mortality, up to 10-year PCa-specific survival rates approaching 98\% [32-34]. Men that have been in an Active Surveillance program showed that metastases were diagnosed in 1 of 200 men at the time of the shift towards invasive therapy [41].

Because non-invasive monitoring based on PSA testing of men with $\mathrm{PCa}$ entail the risk of disease progression, repeat transrectal biopsies are the cornerstone of AS [37, 38]. In a large AS cohort of 757 patients the first repeat biopsy was taken after a median follow-up of 1 year, which led to reclassification of risk in $21 \%$ of patients [40]. However these data should be interpreted with caution since there may have been potential understaging at time of the diagnostic prostate biopsy.

The currently applied inclusion criteria for AS thus may select men with significant disease. Applying a probabilistic selection using a nomogram which includes the clinical parameters lika PSA, T-stage and Gleason score might be of help and may decrease the frequency of misclassification. This was studied within ERSPC using men with screen detected $\mathrm{PCa}$ and having had a radical prostatectomy $(n=1011)$. With indolent PCa defined as a tumour volume less than 0.5 $\mathrm{cc}$, and confined to the prostate, and with no Gleason pattern 4 or 5 , a total of $26 \%$ of men had an indolent $\mathrm{PCa}$. Stricter rule-based and higher thresholds criteria (i.e. a PSAD threshold of $<0.15 \mathrm{ng} / \mathrm{ml} / \mathrm{cc}$ and $<0.10$ $\mathrm{ng} / \mathrm{ml} / \mathrm{cc}$ instead of $<0.20 \mathrm{ng} / \mathrm{ml} / \mathrm{cc}$, a maximum number of positive biopsy cores of 1 instead of 2 , and a maximum percentage of biopsy core tumour involvement of $50 \%$ instead of no threshold) of indolent disease resulted in a higher frequency of indolent disease that was included for radical prostatectomy, up to $61-67 \%$, but at the cost of a decrease in the number of men suitable for AS, down to $2-17 \%$. These 
refinements in selection did not have a significant effect on biochemical progression rates [data coming from ERSPC, oral communication, thesis from $R$. van den Bergh, 2009, ISBN: 978-90-8559-602-8].

Although Active Surveillance appears to be a safe treatment option, longer follow-up of patients with favourable PCa risk profiles is desirable, to optimise and balance the inclusion criteria and criteria to switch to active treatment. One of the largest prospective Active Surveillance studies designed to evaluate these issues is the PRIAS study; a follow-up registry for active surveillance of low-risk prostate cancer, initiated in 2006 [53]. Recent data from the PRIAS study combined with an overview of the literature from the past 3 years showed that risk reclassification on repeat biopsy has occurred in $27 \%$ of men and a switch towards active therapy occurred in $22 \%$ of men under AS [53].

As mentioned earlier, compliance studies on nomogram recommendations have been rarely performed. In one of the first studies to investigate the compliance of urologists and patients with recommendations concerning the probability of indolent PCa based on a risk calculator, and hence treatment choice data showed that AS and active treatment recommendations were followed by $82 \%$ and $71 \%$ of patients, respectively [54]. However $29 \%$ with active treatment recommendations (based on a calculated probability of indolent disease $<70 \%$ ) chose AS instead. The most common reason for non-compliance with active treatment recommendations by urologists was the patient's preference for AS. The threshold set for a recommendation of AS vs. active treatment may be too high for urologists and patients, however the risk calculator proved to be useful for patients in decisionmaking, because AS recommendations were followed by most patients [54].

\section{TREATMENT WITH AS, SWITCHING TO ACTIVE TREATMENT}

PSA kinetics are generally assumed to be indicative of tumor progression and are therefore used in decision-making in men on AS for PCa. A review showed that the evidence concerning the prognostic value of the PSA doubling time (PSA-DT) is sparse, especially in active surveillance, and therefore should be combined with other diagnostic measures as the trigger for repeat prostate biopsies or radical treatment [51]. Therefore nomograms are currently based on Gleason scores, PSA-level kinetics and DRE. In the near future, advanced radiologic imaging and new biologic markers, should help clinicians and patients choose optimal follow-up and treatment pathways. The improvement of non-invasive biomarkers in serum or urine, or radiologic interventions like MRI-guided prostate biopsies to monitor disease progression can possibly alter current AS schemes. The use of genetic markers, such as PCA3 and TMPRSS2-ERG, has the potential to aid disease screening and improve prognostic discrimination [52]. A recent literature review revealed however that most markers have not yet been prospectively validated for providing useful prognostic or predictive information. In the future these clinically diagnostic tests may improve clinical decision making [52].

Besides the currently available static models which include patient and tumor characteristics at baseline, prognostic models should take new information into account that accumulate during follow-up which change the clinical status of the patient. This so-called dynamic risk modelling allows updating the prognosis of a patient according to the evolution of his disease. Updated predictions will allow individualized management of the patients follow-up schemes and enable tailored choices concerning different treatment modalities. Further research should therefore focus on the development of these dynamic models including potential new biomarkers in order to reach an optimal outcome of survival and quality of life.

\section{CONCLUSION}

Systematic PSA-based screening can reduce mortality from PCa. However, currently it coincides with considerable harms, such as unnecessary testing and prostate biopsies and overdiagnosis. With risk stratification tools, we may be able to identify these men who are at increased risk of having a potentially life threatening $\mathrm{PCa}$, and therefore avoid biopsies in men who are not likely to benefit from it. In addition, in men with low risk disease, active surveillance, including dynamic risk stratification at time of inclusion and during follow-up can be considered a management strategy to postpone or even avoid radical treatment, although currently no empirical data exists.

To reduce overdiagnosis and overtreatment of prostate cancer in the current PSA era, individualization and optimization of diagnosis, monitoring and treatment thus is desirable in which nomograms are likely to play an important role. 


\section{REFERENCES}

[1] Jemal A, Bray F, Center MM, et al. Global cancer statistics. CA Cancer J Clin 2011; 61: 69-90. http://dx.doi.org/10.3322/caac.20107

[2] Draisma G, Boer R, Otto SJ, et al. Lead times and overdetection due to prostate-specific antigen screening: estimates from the European Randomized Study of Screening for Prostate Cancer. J Natl Cancer Inst 2003; 95(12): 868-78.

http://dx.doi.org/10.1093/jnci/95.12.868

[3] Schröder FH, Hugosson J, Roobol MJ, et al. Screening and Prostate-Cancer Mortality in a Randomized European Study. N Engl J Med 2009; 360: 1320-8. http://dx.doi.org/10.1056/NEJMoa0810084

[4] Schröder FH, Hugosson J, Roobol MJ, et al. Prostate-Cancer Mortality at 11 Years of Follow-up. N Engl J Med 2012; 366: 981-90. http://dx.doi.org/10.1056/NEJMoa1113135

[5] Hugosson J, Carlsson S, Aus G, Bergdahl S, et al. Mortality results from the Göteborg randomised population-based prostate-cancer screening trial. Lancet Oncol 2010; 11(8): 725-32.

http://dx.doi.org/10.1016/S1470-2045(10)70146-7

[6] Cooperberg MR, Broering JM, Carroll PR. Time trends and local variation in primary treatment of localized prostate cancer. J Clin Oncol 2010; 28(7): 1117-23.

http://dx.doi.org/10.1200/JCO.2009.26.0133

[7] Welch HG, Albertsen PC. Prostate cancer diagnosis and treatment after the introduction of prostate-specific antigen screening: 1986-2005. J Natl Cancer Inst 2009; 101(19): 1325-9.

http://dx.doi.org/10.1093/jnci/djp278

[8] Potosky AL, Davis WW, Hoffman RM, et al. Five-year outcomes after prostatectomy or radiotherapy for prostate cancer: the prostate cancer outcomes study. J Natl Cancer Inst 2004; 96(18): 1358-67. http://dx.doi.org/10.1093/jnci/djh259

[9] Korfage IJ, Essink-Bot ML, Borsboom GJ, et al. Five-year follow-up of health-related quality of life after primary treatment of localized prostate cancer. Int J Cancer 2005; 116(2): 291-6.

http://dx.doi.org/10.1002/ijc.21043

[10] Johansson E, Steineck G, Holmberg L, et al. Long-term quality-of-life outcomes after radical prostatectomy or watchful waiting: the Scandinavian Prostate Cancer Group-4 randomised trial. Lancet Oncol 2011; 12: 891-9. http://dx.doi.org/10.1016/S1470-2045(11)70162-0

[11] Shariat SF, Karakiewicz PI, Roehrborn CG, et al. An updated catalog of prostate cancer predictive tools. Cancer 2008; 113(11): 3075-99 http://dx.doi.org/10.1002/cncr.23908

[12] Shariat SF, Kattan MW, Vickers AJ, et al. Critical review of prostate cancer predictive tools. Future Oncol 2009; 5(10): 1555-84.

http://dx.doi.org/10.2217/fon.09.121

[13] Zhu X, Albertsen PC, Andriole GL, et al. Risk-based prostate cancer screening. Eur Urol 2012; 61: 652-61. http://dx.doi.org/10.1016/j.eururo.2011.11.029

[14] Vickers AJ. Prediction models in cancer care. CA Cancer J Clin 2011; 61: 315-326.

[15] The University of Texas Health Science Center [homepage on the Internet]. San Antonio, TX: The University of Texas Health Science Center at San Antonio Copyright (c) 2010. [updated 2012 April 12; cited 2012 Dec 10]. The Prostate Cancer Prevention Trial Prostate Cancer Risk Calculator (PCPTRC). Available from: http://deb.uthscsa.edu/ URORiskCalc/Pages/uroriskcalc.jsp
Thompson IM, Ankerst DP, Chi C, et al. Assessing prostate cancer risk: results from the Prostate Cancer Prevention Trial. J Natl Cancer Inst 2006; 98: 529-34. http://dx.doi.org/10.1093/inci/dij131

[17] Eyre SJ, Ankerst DP, Wei JT, et al. Validation in a multiple urology practice cohort of the Prostate Cancer Prevention Trial calculator for predicting prostate cancer detection. $\mathrm{J}$ Urol 2009; 182: 2653-8. http://dx.doi.org/10.1016/j.juro.2009.08.056

[18] Hernandez DJ, Han M, Humphreys EB, et al. Predicting the outcome of prostate biopsy: comparison of a novel logistic regression-based model, the prostate cancer risk calculator, and prostate-specific antigen level alone. BJU Int 2009; 103: 609-14. http://dx.doi.org/10.1111/j.1464-410X.2008.08127.x

[19] Nguyen CT, Yu C, Moussa A, Kattan MW, et al. Performance of prostate cancer prevention trial risk calculator in a contemporary cohort screened for prostate cancer and diagnosed by extended prostate biopsy. J Urol 2010; 183: 529-33.

http://dx.doi.org/10.1016/j.juro.2009.10.007

[20] Sunnybrook Health Sciences Centre [homepage on the Internet]. Toronto, ON: Sunnybrook Health Sciences Centre c2013 [updated 2012; cited 2012 Dec 10]: Sunnybrook Prostate Cancer Risk Calculator. Available form: http://sunnybrook.ca/content/?page=OCC prostateCalc

[21] Nam RK, Toi A, Klotz LH, et al. Assessing individual risk for prostate cancer. J Clin Oncol 2007; 25: 3582-88. http://dx.doi.org/10.1200/JCO.2007.10.6450

[22] Nam RK, Kattan MW, Chin JL, et al. Prospective multiinstitutional study evaluating the performance of prostate cancer risk calculators. J Clin Oncol 2011; 29: 2959-64. http://dx.doi.org/10.1200/JCO.2010.32.6371

[23] SWOP - The Prostate Cancer Research Foundation [homepage on the Internet]. Rotterdam, The Netherlands: European Randomized Study of Screening for Prostate Cancer; c2013 [updated 2012; cited 2012 Oct 10]: The Prostate Risk Indicator. Available form: www.prostatecancerriskcalculator.com

[24] Roobol MJ, Steyerberg EW, Kranse R, et al. A risk-based strategy improves prostate-specific antigen-driven detection of prostate cancer. Eur Urol 2010; 57: 79-85. http://dx.doi.org/10.1016/j.eururo.2009.08.025

[25] Kranse R, Roobol M, Schroder FH. A graphical device to represent the outcomes of a logistic regression analysis. Prostate 2008; 68(15): 1674-80. http://dx.doi.org/10.1002/pros.20840

[26] Steyerberg EW, Roobol MJ, Kattan MW, et al. Prediction of indolent prostate cancer: validation and updating of a prognostic nomogram. J Urol 2007; 177: 107-12. http://dx.doi.org/10.1016/i.juro.2006.08.068

[27] Oliveira M, Marques V, Carvalho AP, et al. Head-to-head comparison of two online nomograms for prostate biopsy outcome prediction. BJU Int 2011; 107: 1780-3.

http://dx.doi.org/10.1111/j.1464-410X.2010.09727.x

[28] Cavadas V, Osorio L, Sabell F, et al. Prostate Cancer Prevention Trial and European Randomized Study of Screening for Prostate Cancer risk calculators: a performance comparison in a contemporary screened cohort. Eur Urol 2010; 58: 551-8. http://dx.doi.org/10.1016/j.eururo.2010.06.023

[29] Trottier G, Roobol MJ, Lawrentschuk N, et al. Comparison of risk calculators from the Prostate Cancer Prevention Trial and the European Randomized Study of Screening for Prostate Cancer in a contemporary Canadian cohort. BJU Int 2011; 108: 237-44 http://dx.doi.org/10.1111/j.1464-410X.2011.10207.x 
[30] Van Vugt HA, Roobol MJ, Kranse R, et al. Prediction of prostate cancer in unscreened men: external validation of a risk calculator. Eur J Cancer 2011; 47: 903-9. http://dx.doi.org/10.1016/j.ejca.2010.11.012

[31] Cooperberg MR, Broering JM, Kantoff PW, et al. Contemporary trends in low risk prostate cancer: risk assessment and treatment. J Urol 2007; 178: 14-9. http://dx.doi.org/10.1016/.juro.2007.03.135

[32] Klotz L, Zhang L, Lam A, et al. Clinical results of long-term follow-up of a large, active surveillance cohort with localized prostate cancer. J Clin Oncol 2010; 28(1): 126-31. http://dx.doi.org/10.1200/JCO.2009.24.2180

[33] Stattin P, Holmberg E, Johansson JE, et al. Outcomes in localized prostate cancer: National Prostate Cancer Register of Sweden follow-up study. J Natl Cancer Inst 2010; 102(13): 950-8.

http://dx.doi.org/10.1093/jnci/dja154

[34] Bul M, van den Bergh RC, Zhu X, et al. Outcomes of initially expectantly managed patients with low or intermediate risk screen-detected localized prostate cancer. BJU Int 2012; 110: $1672-7$.

http://dx.doi.org/10.1111/j.1464-410X.2012.11434.x

[35] Heidenreich A, Bellmunt J, Bolla M, et al. EAU guidelines on prostate cancer. Part 1: screening, diagnosis, and treatment of clinically localised disease. Eur Urol 2011; 59(1): 61-71. http://dx.doi.org/10.1016/j.eururo.2010.10.039

[36] Roemeling S, Roobol MJ, De Vries $\mathrm{SH}$, et al. Active surveillance for prostate cancers detected in three subsequent rounds of a screening trial: characteristics, PSA doubling times, and outcome. Eur Urol 2007; 51: 1244-1250. http://dx.doi.org/10.1016/j.eururo.2006.11.053

[37] Ross AE, Loeb $S$, Landis $P$, et al. Prostate-specific antigen kinetics during follow-up are an unreliable trigger for intervention in a prostate cancer surveillance program. J Clin Oncol 2010; 28(17): 2810-6.

http://dx.doi.org/10.1200/JCO.2009.25.7311

[38] Whitson JM, Porten SP, Hilton JF, et al. The relationship between prostate specific antigen change and biopsy progression in patients on active surveillance for prostate cancer. J Urol 2011; 185(5): 1656-60.

http://dx.doi.org/10.1016/j.juro.2010.12.042

[39] Etzioni R, Gulati R, Tsodikov A, et al. The prostate cancer conundrum revisited: Treatment changes and prostate cancer mortality declines. Cancer 2012; 118(23): 5955-63. http://dx.doi.org/10.1002/cncr.27594

[40] Bul M, van den Bergh RC, Rannikko A, et al. Predictors of unfavourable repeat biopsy results in men participating in a prospective active surveillance program. Eur Urol 2012; 61(2): 370-7.

http://dx.doi.org/10.1016/j.eururo.2011.06.027

[41] Van den Bergh RC, Roemeling S, Roobol MJ, et al. Prospective validation of active surveillance in prostate cancer: the PRIAS study. Eur Urol 2007; 52: 1560-63. http://dx.doi.org/10.1016/j.eururo.2007.05.011

[42] Raaijmakers R, Kirkels WJ, Roobol MJ, et al. Complication rates and risk factors of 5802 transrectal ultrasound-guided sextant biopsies of the prostate within a population-based screening program. Urology 2002; 60: 826-30. http://dx.doi.org/10.1016/S0090-4295(02)01958-1
[43] Loeb S, van den Heuvel S, Zhu X, et al. Infectious complications and hospital admissions after prostate biopsy in a European randomized trial. Eur Urol 2012; 61(6): 11104.

http://dx.doi.org/10.1016/j.eururo.2011.12.058

[44] Roobol MJ, Zhu X, Schröder FH, et al. A Calculator for Prostate Cancer Risk 4 Years After an Initially Negative Screen: Findings from ERSPC Rotterdam. Eur Urol 2012. [Published online 20 July 2012].

[45] Xia J, Trock BJ, Cooperberg MR, et al. Prostate Cancer Mortality following Active Surveillance versus Immediate Radical Prostatectomy. Clin Cancer Res 2012; 18(19): 5471 . 78.

http://dx.doi.org/10.1158/1078-0432.CCR-12-1502

[46] Ankerst DP, Boeck A, Freedland SJ, et al. Evaluating the PCPT risk calculator in ten international biopsy cohorts: results from the Prostate Biopsy Collaborative Group. World J Urol 2012; 30(2): 181-7.

\section{http://dx.doi.org/10.1007/s00345-011-0818-5}

[47] Van Vugt HA, Kranse R, Steyerberg EW, et al. Prospective validation of a risk calculator which calculates the probability of a positive prostate biopsy in a contemporary clinical cohort. Eur J Cancer 2012; 48(12): 1809-15. http://dx.doi.org/10.1016/j.ejca.2012.02.002

[48] Van Vugt HA, Roobol MJ, Busstra M, et al. Compliance with biopsy recommendations of a prostate cancer risk calculator. BJU Int 2012; 109(10): 1480-8. http://dx.doi.org/10.1111/j.1464-410X.2011.10611.x

[49] Van den Bergh RC, Roemeling $S$, Roobol MJ, et al. Prospective validation of active surveillance in prostate cancer: the PRIAS study. Eur Urol 2007; 52(6): 1560-3. http://dx.doi.org/10.1016/j.eururo.2007.05.011

[50] Van den Bergh RC, Roemeling S, Roobol MJ, et al. Outcomes of men with screen-detected prostate cancer eligible for active surveillance who were managed expectantly. Eur Urol 2009; 55(1): 1-8. http://dx.doi.org/10.1016/j.eururo.2008.09.007

[51] Van den Bergh RC, Roemeling S, Roobol MJ, et al. Prostatespecific antigen kinetics in clinical decision-making during active surveillance for early prostate cancer - a review. Eur Urol 2008; 54(3): 505-16. http://dx.doi.org/10.1016/j.eururo.2008.06.040

[52] Choudhury AD, Eeles R, Freedland SJ, et al. The role of genetic markers in the management of prostate cancer. Eur Urol 2012; 62(4): 577-87. http://dx.doi.org/10.1016/j.eururo.2012.05.054

[53] Bangma $\mathrm{CH}$, Bul M, Roobol M. The Prostate cancer Research International: Active Surveillance study. Curr Opin Urol 2012; 22(3): 216-21. http://dx.doi.org/10.1097/MOU.0b013e328351dcc7

[54] Van Vugt HA, Roobol MJ, van der Poel HG, et al. Selecting men diagnosed with prostate cancer for active surveillance using a risk calculator: a prospective impact study. BJU Int 2012; 110(2): 180-7.

http://dx.doi.org/10.1111/j.1464-410X.2011.10679.x

[55] Stricker PD, Frydenberg M, Kneebone A, et al. Informed prostate cancer risk-adjusted testing: a new paradigm. BJU Int 2012; 110(Suppl 4): 30-4. http://dx.doi.org/10.1111/j.1464-410X.2012.11621.x 